\title{
Environmental invariants in the representation of motion: Implied dynamics and representational momentum, gravity, friction, and centripetal force
}

\author{
TIMOTHY L. HUBBARD \\ University of Arizona, Tucson, Arizona
}

\begin{abstract}
Memory for the final position of a moving target is often shifted or displaced from the true final position of that target. Early studies of this memory shift focused on parallels between the momentum of the target and the momentum of the representation of the target and called this displacement representational momentum, but many factors other than momentum contribute to the memory shift. A consideration of the empirical literature on representational momentum and related types of displacement suggests there are at least four different types of factors influencing the direction and magnitude of such memory shifts: stimulus characteristics (e.g., target direction, target velocity), implied dynamics and environmental invariants (e.g., implied momentum, gravity, friction, centripetal force), memory averaging of target and nontarget context (e.g., biases toward previous target locations or nontarget context), and observers' expectations (both tacit and conscious) regarding future target motion and target/context interactions. Several theories purporting to account for representational momentum and related types of displacement are also considered.
\end{abstract}

An observer's memory regarding the final position of a previously perceived target is often distorted in ways consistent with the operation of invariant physical principles. For example, an observer indicating the remembered final orientation of a previously perceived rotating target will often indicate an orientation that is shifted slightly forward in the direction of target rotation from the actual final orientation of the target. One early theory to explain this forward shift drew upon an analogy between the momentum of the physical target and the momentum of the mental representation of the target, and referred to this forward shift as representational momentum (Freyd \& Finke, 1984). Momentum is just one of several environmentally invariant factors that distort memory, however; in this discussion, therefore, the term representational momentum will be restricted to discussions of the influence of target momentum. The more general terms displacement and shift will be used to describe the distortions produced by other environmentally invariant factors or by noninvariant factors and the interaction of those other factors with each other and with representational momentum.

The discussion presents a roughly chronological exposition of how our understanding of displacement has grown beyond a concern with momentum and the concrete momentum metaphor and into a broader investigation of the physical principles that have been invariant within human evolutionary experience (e.g., gravity, fric-

The author thanks two anonymous reviewers for helpful comments. Correspondence should be addressed to T. Hubbard, who is now at the Department of Psychology, Texas Christian University, Fort Worth, TX 76129. tion, centripetal force) and that appear to influence our representation of physical systems in systematic ways. Influences of individual experience and expectation on displacement will also be examined, and attempts to incorporate the effects of both environmental invariants and individual expectations on displacement will elaborate the bridge between Gibsonian and representational theories first suggested by Shepard (1984). Similarities between the displacements attributable to environmental invariants and the recent findings of boundary extension of pictorial memory will be suggested. A reconciliation will be proposed of the seeming contradiction between claims that physically invariant principles have been incorporated into either individual representations or our representational system and claims from studies on socalled naive physics that people do not have veridical intuitions regarding the behavior of physical systems. Lastly, directions and constraints for future formal modeling of displacement phenomena will be suggested.

\section{Representational Momentum}

In the first demonstration of representational momentum, Freyd and Finke (1984) presented observers with four computer-animated sequential presentations of a rectangular target. The first three presentations were referred to as the inducing stimuli, and the fourth presentation was referred to as the probe or test stimulus. The inducing stimuli and the probe were presented at the same concentric location but varied in orientation. The observers' task was to judge whether the probe was presented at the same orientation as the previously shown final inducing stimulus. If the orientations of consecutive inducing stimuli implied rotation in either a consistent clockwise or a consistent counterclockwise direction, 
observers were more likely to respond same if the orientation of the probe was slightly beyond the orientation of the final inducing stimulus than if the orientation of the probe was slightly behind the orientation of the final inducing stimulus. In other words, observers were more likely to respond same if the probe was rotated slightly forward from the orientation of the final inducing stimulus than if the probe was rotated slightly backward from the orientation of the final inducing stimulus. This asymmetry was not observed if motion of the inducing stimuli did not imply consistent motion in a single direction. In a follow-up report, Freyd and Finke (1985) demonstrated that the magnitude of representational momentum increased with increases in target velocity.

Freyd and Finke $(1984,1985)$ presented only one target on each trial, so the direction and velocity of observers' pursuit eye movements presumably paralleled target motion. In order to minimize any contributions of eye movements to representational momentum and examine whether representational momentum could also be found with larger and more complex configurations consisting of multiple targets, Finke and Freyd (1985) presented observers with inducing stimuli consisting of patterns of dots in which each dot moved in a different direction. By having each of the multiple targets within a single display move in a different direction, it was hoped that any eye movement artifact would be eliminated, because the observers' eyes could not simultaneously track targets moving in different directions. Forward probes were more difficult to reject than backward probes, but no effects of target velocity were found. Finke, Freyd, and Shyi (1986) subsequently replicated the Finke and Freyd (1985) experiment using a wider range of probe configurations, and found both standard representational momentum and velocity effects. Finke et al. (1986) also reported that greater positive acceleration led to greater magnitudes of representational momentum, and that the magnitude of representational momentum depended primarily on the final instantaneous velocity of the target.

It was possible that the forward shifts found by Freyd and Finke (1984) and Finke et al. (1986) were obtained because observers were mistakenly attempting to predict the subsequent position or configuration of the target rather than judging whether the probe was at the same position or in the same configuration as the final inducing stimulus. Finke and Shyi (1988) used dot-pattern displays similar to those used by Finke et al. (1986) to further examine this possibility by comparing the shift in the distributions of observers' responses in a memory task with the shift in the distributions of observers' responses in a prediction task. In the memory task, observers judged whether the configuration of dots in the probe was the same as the configuration of dots in the final inducing stimulus; in the prediction task, observers judged whether the configuration of dots in the probe matched what would have been the next step in the inducing sequence had the inducing sequence continued. In general, in the memory task, the distributions of observers' responses were shifted forward, but in the prediction task, the dis- tributions of observers' responses were shifted backward. These differences in the direction of shift between the memory task and the prediction task supported the notion that representational momentum was not produced by observers' predictions of the subsequent configuration of the inducing stimuli.

In a similar set of investigations, Hubbard and Bharucha (1988) presented observers with animated displays in which a single small circular target moved either horizontally or vertically across a cathode-ray tube (CRT) monitor. Unlike the discrete and clearly separated inducing stimuli used by Freyd and Finke $(1984,1985)$, the target stimuli presented by Hubbard and Bharucha exhibited smooth and continuous apparent motion. Different target velocities were used, and the target would vanish without warning. After the target vanished, the observers used a computer mouse to position the cursor over where they believed the vanishing point of the target to have been, and by clicking a button on the mouse the screen coordinates of the judged vanishing point (i.e., the cursor) were recorded and could be directly compared with the screen coordinates of the actual vanishing point. This cursor-positioning response method simplified investigation by offering a much more direct examination of memory for target position than was possible with the use of probes. Additionally, the cursor-positioning response method allowed for the possibility that memory might be shifted in directions other than along the path of motion.

The judged vanishing point was systematically displaced from the true vanishing point along both the axis of motion (referred to as $M$ displacement; the $x$ axis for horizontal motion and the $y$ axis for vertical motion) and the axis orthogonal to motion (referred to as $O$ displacement; the $y$ axis for horizontal motion and the $x$ axis for vertical motion). The $M$ displacement values revealed that memory for the target was shifted in the direction of implied motion; furthermore, the magnitude of $M$ displacement increased with increases in target velocity. This pattern was fully consistent with the representational momentum data reported by Freyd, Finke, and their colleagues. Additionally, horizontal motion led to larger forward $M$ displacement than did vertical motion, and descending motion led to larger forward $M$ displacement than did ascending motion. The $O$ displacement values revealed that memory for targets moving horizontally was also shifted below the path of motion, and that memory for targets moving vertically was not systematically shifted to either the left or the right of the path of motion.

The effect of time upon the magnitude of representational momentum was examined by Freyd and Johnson (1987) in a series of experiments in which the retention interval between the offset of the final inducing stimulus and the onset of the probe was varied. In general, the magnitude of representational momentum increased rapidly as the retention interval increased to approximately $300 \mathrm{msec}$ and then gradually decreased as the retention interval increased. Furthermore, the magnitude of representational momentum peaked and fell off more quickly 
for stimuli moving at faster implied velocities. Freyd and Johnson proposed that this pattern resulted from a fast initial forward shift which was then nullified by memory averaging toward the central tendency of the inducing stimuli. In other words, an initial forward shift that resulted from representational momentum was then offset by a subsequent backward shift that resulted from an averaging in memory of the spatial coordinates of the final inducing stimulus with the spatial coordinates of the prior inducing stimuli. A roughly comparable time course for representational momentum with auditory (tone) stimuli was subsequently reported by Freyd, Kelly, and DeKay (1990). Such a short time course for representational momentum was surprising in light of results from both Finke and Freyd (1985) and Hubbard and Bharucha (1988), who reported that observers had more difficulty rejecting forward distractors even when the retention interval was $2 \mathrm{sec}$.

Whether representational momentum is exhibited also depends upon the maintenance of a single target identity across the presentation of the inducing and probe stimuli. In a number of experiments, Kelly and Freyd (1987) presented discrete inducing and probe stimuli in which the inducing stimuli could differ from each other in size, shape, or internal markings and the probes could similarly differ from the inducing stimuli. If all of the stimuli differed, no representational momentum was observed. If there were minor changes in target shape or contour across the inducing and probe stimuli and the changes were consistent with a single object undergoing a continuous transformation (e.g., a rectangle growing progressively narrower), representational momentum was obtained unless the shape of the final inducing stimulus was a very prototypical shape (e.g., a square). If the contour of the inducing stimuli was constant but internal markings differed, accuracy data indicated representational momentum but response time data did not indicate representational momentum. Overall, the data suggested that in order for representational momentum to be clearly and consistently manifested, the observers had to assume that the displays depicted a single object undergoing a consistent type of change or transformation. If this assumption was violated (e.g., when the size, shape, or markings of each of the inducing stimuli were radically different), representational momentum was not produced because the visual system treated each of the inducing stimuli as representing a different stationary object.

Representational momentum is also influenced by the direction of target motion. Although the direction of rotation (i.e., clockwise or counterclockwise) has not been consistently shown to influence the magnitude of representational momentum, the direction of translation has been consistently shown to influence the magnitude of representational momentum. Hubbard and Bharucha (1988) reported that forward $M$ displacement was larger for targets traveling horizontally than it was for targets traveling vertically. Hubbard (1990) replicated this asymmetry and also found that targets traveling along oblique paths exhibited an average displacement intermediate in mag- nitude to the average displacement magnitudes of targets traveling along horizontal paths and targets traveling along vertical paths. In essence, the closer the path of motion was to the horizontal axis, the larger was the average displacement. Hubbard and Bharucha (1988; also Hubbard, 1990) also consistently found asymmetries within vertical motion such that descending motion produced larger forward $M$ displacement than did ascending motion, but they did not find consistent asymmetries in the magnitude of $M$ displacement between horizontal motion of targets moving toward the left and targets moving toward the right. Neither Freyd et al. (1990) nor Hubbard (1995a) found consistent differences in the magnitude of representational momentum between ascending and descending auditory pitch sequences.

Halpern and Kelly (1993) examined representational momentum for horizontally moving targets and reported that targets moving toward the right exhibited consistently larger forward (smaller backward) displacements than targets moving toward the left. This difference between rightward and leftward motion was relatively insensitive to target velocity or to whether the target was depicted as a mechanical or a biological entity. Halpern and Kelly also reported greater forward displacement for targets in the left half of visual space, and this asymmetry is consistent with that found by White, Minor, Merrell, and Smith (1993), who reported greater representational momentum for probes presented in the left visual field. White et al. also noted that the left field advantage was stronger when the interstimulus interval between the disappearance of the final inducing stimulus and the appearance of the probe was relatively short, and they proposed that representational momentum is greater in the right hemisphere (left visual field) than it is in the left hemisphere (right visual field).

\section{Evidence Against Low-Level Explanations}

Potential low-level explanations for representational momentum involved sensory aspects of the display and appealed to factors such as motion aftereffects, pattern afterimages (due to slow screen decay), and eye movements. The motion aftereffects and pattern afterimages hypotheses could be easily dismissed; as pointed out by Finke and Freyd (1985) and Hubbard and Bharucha (1988), motion aftereffects and pattern afterimages would in most cases have been in the direction opposite to the displacement patterns actually found and would have biased against displacement in the forward direction. The eye movement hypothesis could not be as easily dismissed, however. The eyes are physical structures, and physical structures acquire inertia as they move. It seemed plausible that observers' eyes acquired inertia as they tracked the targets, and that when the targets vanished, that inertia prevented the eyes from stopping immediately, but carried them (and the fixation point) forward some distance beyond the vanishing point. Furthermore, targets moving at faster velocities would have acquired greater inertia, and so the eyes would have been carried forward a greater distance after the target vanished. This 
type of explanation seemed to account for representational momentum if it were granted that memory for the vanishing point corresponded to where the eyes stopped rather than to where the target vanished.

Evidence against the eye movement hypothesis came from three main sources. First, representational momentum has been found with auditory stimuli (Freyd et al., 1990; Hubbard, 1995a), and it is not clear how eye movements could contribute to representational momentum in a nonvisual modality such as audition, or what the auditory equivalent of eye movements would be in creatures with fixed pinnae, such as humans. Second, studies presenting multiple visual targets in which each target moved in a different direction (Finke et al., 1986; Finke \& Shyi, 1988) rendered it highly unlikely that observers' eye movements would have closely paralleled target motion for every target (even if observers engaged in an extremely rapid time-sharing of attentional resources across the different targets). Third, the displacement patterns for visually moving stimuli have been shown to reflect the anticipated, rather than the actual, direction of target motion (Bharucha \& Hubbard, 1989; Hubbard \& Bharucha, 1988). Any role of anticipation or expectation moves the phenomenon from a purely sensory realm into a more cognitive realm.

Many early experiments on displacement confounded the direction of anticipated target motion and the direction of actual target motion at the time the target vanished, and so forward displacement may have reflected either the physical inertia of the eyes or a more cognitive expectation of future target location. Hubbard and Bharucha (1988) separated the anticipated direction of motion from the current direction of motion by presenting a horizontally or vertically moving target inside a surrounding stationary frame. The target would approach, collide with, and then bounce off the inner surface of the frame. Each target would bounce from one to five times before the target and frame would simultaneously vanish. For targets that vanished just prior to collision or at the moment of collision, the actual path of motion and the anticipated path of motion would be in different directions; for targets that vanished just after collision and the subsequent bounce, the actual path of motion and the anticipated path of motion would be in the same direction. If the target vanished just prior to collision or at the moment of collision, judged vanishing point was displaced behind the true vanishing point, but if the target vanished just after collision and the subsequent bounce, judged vanishing point was displaced beyond the true vanishing point. In all cases, displacement was in the direction of anticipated motion rather than in the direction of actual motion at the time the target vanished, and so a lower level sensory explanation was ruled out in favor of an explanation based on a higher order cognitive expectation.

In a similar study, Bharucha and Hubbard (1989) presented observers with an obliquely moving target within a stationary square frame, and the use of oblique motion resulted in a greater spatial separation of the coordinates of the precollision and postcollision vanishing points and a greater spatial separation between the anticipated direction of target motion at each vanishing point. As before, the target would approach, collide with, and bounce off the inner surface of the frame, and each target would bounce between one and five times before the target and frame simultaneously vanished. As before, the judged vanishing point was displaced in the anticipated direction of motion rather than in the actual direction of motion at the time the target vanished. Subsequent control experiments removed target motion from the display and briefly presented either the frame and a stationary target or just a stationary target, and the stationary targets were presented at the same screen coordinates used for the precollision, collision, and postcollision vanishing points in the earlier experiment; no differences in displacement patterns between stationary targets presented at locations corresponding to the pre- and postcollision vanishing points were observed. Even if subsequent studies show that observers' eye movements in this paradigm move in the direction of anticipated motion, there still must be some higher cognitive process driving the eye movements; therefore, not only did these studies demonstrate the importance of anticipation of future target motion in determining the pattern of displacement, they also allowed eye movements to be ruled out as the sole explanation for displacement.

\section{From the Momentum Metaphor to Spatiotemporal Coherence}

Even before low-level explanations appealing to motion aftereffects, pattern afterimages, and eye movements were ruled out, a more cognitive explanation based on an analogy between physical momentum and representational momentum was being articulated. Finke et al. (1986; also Finke \& Freyd, 1985) suggested that shifts in visual memory for remembered position occur because there is a natural tendency to mentally extrapolate the motion of a target stimulus into the future. This tendency occurs because such extrapolation allows anticipation of the future position of the target and allows better regulation of bodily movements. This extrapolation process possesses a kind of inertia (i.e., representational momentum), and as a consequence of this inertia the mental extrapolations, just like moving physical objects, cannot be instantly halted but must continue for some time after a person first begins to stop them. If a physical object is in motion and a force is applied to stop that motion, the object cannot stop immediately but will, rather, coast some distance due to the momentum acquired during its motion. Similarly, the mental representation of an object in motion possesses its own form of momentum and cannot stop immediately. The mental representation, like the physical object it represents, will continue to coast some distance after a stopping force has been applied (see also Finke \& Freyd, 1989). Also, just as the momentum of a moving object increases as the velocity of that object increases and thus makes that object more difficult to stop, 
so too do the mental extrapolations become more difficult to stop as the velocity of the inducing stimuli increases.

The analogy between physical momentum and representational momentum was not a complete analogy, and Finke et al. (1986) suggested that differences between the two types of momentum do exist. First, presumably momentum for a physical target at time $T_{3}$ would be independent of its prior direction of motion between time $T_{1}$ and time $T_{2}$, yet the differences in representational momentum between consistent and inconsistent motion conditions show that the prior direction of motion from time $T_{1}$ to time $T_{2}$ does influence representational momentum at time $T_{3}$ (Freyd \& Finke, 1984). Second, momentum for a physical target is exhibited along a highly constrained physical pathway, but Finke et al. proposed that the correspondence between physical momentum and representational momentum is limited to how the mental extrapolations are stopped, and not to the particular pathways that the extrapolation might follow. Finke and Freyd (1989) expanded on this last notion by comparing representational momentum to a train; more specifically, the mental representation would be like a train running along a track: While one could easily change the direction of the train by switching the track ahead of it, the momentum of the train would still carry it along the chosen track.

Finke et al. (1986) suggested that it should be possible to obtain representational momentum for any type of implied transformation and any type of stimulus, but more recently, Freyd $(1987,1992,1993)$ suggested that representational momentum could be obtained only if target stimuli were drawn from a continuous dimension (e.g., orientation, location) and could not be obtained if target stimuli were drawn from a discrete dimension (e.g., integers). According to Freyd's more recent hypothesis, it is the underlying nature of the stimulus dimension that determines whether or not representational momentum occurs, and so differences in the surface form of the target (e.g., whether the target is presented as a series of discrete inducing stimuli [implied motion] or as a smoothly changing stimulus [apparent motion]) should not influence whether or not representational momentum occurs. This hypothesis was recently supported by similar patterns of displacement for auditory stimuli being found when the inducing stimuli were presented as either smooth pitch glides or as sequences of discrete pitches (Hubbard, 1995a). Similarly, the forward displacement observed with both discrete visual implied motion (e.g., Finke et al., 1986; Freyd \& Finke, 1984) and smooth visual apparent motion (e.g., Hubbard, 1990; Hubbard \& Bharucha, 1988) suggests that differences in the surface form of target presentation do not determine whether representational momentum for a given dimension is observed.

The momentum metaphor developed by Finke, Freyd, and their collaborators suggested that representational momentum exists because the principles of momentum have been incorporated or internalized within the repre- sentational system. Furthermore, this incorporation or internalization occurred because the laws of momentum have been invariant throughout human experience. The idea that principles that had been invariant within human experience could have become incorporated within our representational system echoed the proposals of Shepard (1984) in his attempts to bridge Gibsonian and representational views of mind. Shepard proposed that many cognitive activities (e.g., perceiving, imaging, thinking, and dreaming) are guided by internalizations of the invariant constraints present in the world and that principles governing the physical behavior of the universe could have become internalized within minds that operate within that universe (see also Shepard, 1994). Shepard's speculations may be easily adapted to an explanation of representational momentum: Given that the properties of physical momentum have been invariant over the course of human evolution on this planet, an organism that could exploit those regularities and mentally extrapolate the path of motion of an object in ways consistent with the laws of momentum would have an advantage in survival and be more likely to reproduce. Representational momentum may therefore have been incorporated into our system of representation because it reflects a practical property of the world and so provides us with a selective advantage.

Freyd $(1987,1992,1993)$ has subsequently broadened her thinking beyond momentum and the momentum metaphor and suggested that the human system of mental representation possesses spatiotemporal coherence. Just as physical momentum is a property of physical objects that exist in a world with spatiotemporal coherence, representational momentum is a property of mental representations that exist in a mental world (i.e., mental model) with spatiotemporal coherence. Mental representations are not simply static storage structures acted upon by separate cognitive processes; rather, mental representations may emerge in part from the execution of cognitive processes and contain dynamic aspects corresponding to the forces involved or reflected in the cognitive processes themselves. Furthermore, the dynamic aspects of at least some mental representations mandate that time is represented necessarily and intrinsically within the representation. The requirement of spatiotemporal coherence requires that the representation of time in such a dynamic representation be both continuous (i.e., analog) and directional (i.e., moving only in the forward direction). The spatiotemporal coherence notions of Freyd address not just representational momentum but also the broader issues of the implied dynamics in additional domains such as aesthetic appreciation of art (e.g., painting, sculpture), comprehension of handwritten letters, and biological constraints on apparent motion (see Freyd, 1992, 1993).

Freyd and her colleagues, as well as other researchers, have presented a variety of evidence suggesting that the implied dynamics in a static display can influence memory for the display. For example, Freyd (1983) presented observers with pairs of frozen-action photographs of scenes 
in which motion or action was occurring (e.g., waves crashing on a beach, a person jumping). Observers took longer to identify the second photograph as being different from the first photograph if the two photographs were shown in real-world temporal order. More recently, Futterweit and Beilin (1994) presented elementary schoolchildren and adults with similar pairs of frozen-action photographs and with pairs of photographs that depicted static scenes. With the frozen-action pairs, observers made significantly more errors in identifying the second photograph as different from the first photograph if the photographs were shown in real-world temporal order, but with the static pairs, no effects of presentation order were found. In the frozen-action pairs presented by both Freyd (1983) and Futterweit and Beilin (1994), memory for the first photograph was shifted in the direction of the motion or action, an asymmetry suggesting that the observers were influenced by the dynamic information in the scenes.

Freyd, Pantzer, and Cheng (1988) presented an inducing stimulus consisting of a line drawing of a box resting on top of a compressed spring and probe stimuli consisting of drawings of springs (in isolation) in which the amount of compression was varied. Memory for the spring was shifted in the predicted direction as observers were more likely to accept a probe in which the boxless spring was less compressed. In a similar experiment, Freyd et al. (1988) presented an inducing stimulus consisting of a line drawing of a potted plant hanging from a hook or resting on a table and probe stimuli consisting of drawings of the potted plant without the hook or table. Memory was again shifted in the predicted direction, as observers were more likely to accept a probe in which the potted plant was drawn slightly lower on the screen than a probe in which the potted plant was drawn slightly higher on the screen. In both the spring and potted plant experiments, memory was shifted in ways consistent with the underlying dynamics of the display, because a spring would be less compressed in the absence of a weight and a potted plant would fall without a hook or table to support its weight.

\section{Representational Gravity}

Both Shepard's notion of the internalization of environmental invariants and Freyd's theory of spatiotemporal coherence suggest that factors that are invariantly present with objects should also be found in the mental representation of those objects. One such invariant that exerts a large influence on moving objects and that has presumably been present throughout all of human evolutionary history is gravity, and consideration of this led Hubbard $(1990,1995 \mathrm{~b})$ to suggest that an analogue of gravity or gravitational attraction might be internalized or otherwise incorporated into our representational system and our representations of individual objects. If a gravitational analogue is indeed incorporated into our representation of an object, it could be predicted that memory for that object's location would be displaced in the direction of implied gravitational attraction. Consis- tent with this view, Shiffrar and Shepard (1991) have also speculated that the invariance of a unique gravitational axis may have resulted in the effects of gravity and its concomitants having become internally represented.

Evidence that an analogue of gravity might be incorporated into our representational system in a way similar to the apparent incorporation of an analogue of momentum was observed in Hubbard and Bharucha's (1988) findings (replicated in Hubbard, 1990) that targets moving horizontally were also displaced downward below the axis of motion and that the forward $M$ displacement of descending targets was larger than the forward $M$ displacement of ascending targets. These patterns are consistent with the existence of an internalized gravitational component in mental representation, because an unpowered object moving horizontally drops along a parabolic curve, a falling object accelerates as it falls, and a rising physical object (that does not attain orbital or escape velocity) decelerates and eventually falls. The targets in these experiments were featureless black circles, and so the effects of gravity (and momentum) could not have arisen from schematic or other knowledge activated by recognition of the target's specific content or identity. Obtaining these gravity and momentum effects in the absence of schematic knowledge dependent on target identity is consistent with the hypothesis that the influences of implied gravity and implied momentum are very basic or intrinsic to representation and do not necessarily require information about the target per se.

Effects of physical gravity are not limited to moving physical objects, but are also observed on stationary physical objects, and so if memory does incorporate an analogue of gravity, we should also expect to see effects of implied gravity on memory for stationary targets. The finding of Freyd et al. (1988) discussed earlier-that a line drawing of a potted plant depicted as hanging from a hook or resting on a table was remembered as being slightly lower when memory for the location of the unsupported object was probed - provides evidence of just such a representational gravity. The influence of implied gravity, like the influence of implied momentum, should be found for all types of targets regardless of the content (i.e., identity) of the target. In order to minimize any possible penetration of target content, Hubbard (1995d) briefly presented observers with stationary featureless black squares and with probes that were either slightly higher or lower on the screen. Memory for the squares was shifted downward, and this pattern was consistent with the hypothesis that the influence of implied gravity was very basic or intrinsic to representation and did not require activation of information concerning target identity or scene-like context.

Evidence of representational gravity was also found in an experiment in which Bertamini (1993) presented observers with an inducing stimulus consisting of a line drawing of a circular target midway along an inclined plane. When memory for the location of the target was probed, the observers were more likely to accept probes in which the targets were located farther down the in- 
clined plane (i.e., displaced in the direction of implied gravitational attraction) for inclined planes with the steepest $\left(60^{\circ}\right)$ slope. Failure to find displacement down the inclined plane for the shallower slopes is not necessarily inconsistent with the gravity-analogue hypothesis when we consider that physical objects are more likely to slide down an inclined plane as the angle or slope of the plane increases and gravitational attraction overcomes friction between the object and the surface of the plane; perhaps the implied friction may have been sufficient to overcome the implied gravity on a shallower inclined plane (and thus the target representation did not shift), but insufficient to overcome the implied gravity on a steeper inclined plane (and thus the target representation did shift).

\section{Representational Friction}

Although Bertamini (1993) did not offer such an interpretation, it is possible to interpret his results as demonstrating an interaction between the effects of representational gravity and representational friction. Friction would seem to be a reasonable candidate for an environmentally invariant force that may have become incorporated into our representational system, because when a moving physical object collides with or slides along a surface, some degree of friction or resistance is invariably present. Although Finke et al. (1986) suggested that friction may have some influence on representational momentum, the type of friction they referred to was an opposing force, a cognitive resistance, that observers applied in order to stop the forward extrapolation process. Such a cognitive resistance involves a friction that is separate from the representation and that an observer might apply to the mental representation in order to halt representational momentum; in essence, Finke et al.'s notion of cognitive resistance refers to the mental brakes applied to the representation of the stimulus in order to halt the forward extrapolation process. Friction as an invariant in the world, however, might not be an external controller of the mental representation, but might rather be an inherent and incorporated part of the mental representation itself.

Evidence suggestive of representational friction was seen in the final experiment in Hubbard and Bharucha (1988). A horizontally moving target approached a stationary barrier, and would either vanish before colliding with the barrier or vanish after colliding with and crashing through the barrier. Forward $M$ displacements at immediately pre- and postcollision vanishing points were greatly reduced from forward $M$ displacement at a vanishing point well prior to the immediately precollision vanishing point, and forward $M$ displacement at the collision vanishing point was reduced even further. In Hubbard (1995b), a similar horizontally moving target crashed through a vertical barrier of varying thickness and $M$ displacement was reduced when the vanishing point was at a collision or postcollision location; furthermore, postcollision $M$ displacement was reduced more with thicker barriers. One possible interpretation of these data is that observers' representations incorporated an element analogous to friction that diminished representational momentum much like physical friction diminishes physical momentum.

Additional experiments in Hubbard (1995b) extended the temporal and spatial duration of implied friction by having a horizontally moving target slide along the upper and/or lower edges of much larger stationary objects below and/or above the path of target motion. If the target slid along the upper or lower edge of a single object, forward $M$ displacement decreased. If the target slid between the upper and lower edges of two objects, forward $M$ displacement decreased even further. Thus, the magnitude of forward displacement decreased with increases in implied friction, a pattern fully consistent with the existence of an internalized friction analogue. Unexpectedly, implied friction on the top or bottom edges of a horizontally moving target also attenuated the customary effect of velocity on $M$ displacement. Such an attenuation was not initially predicted by either Shepard's (1984) notion of internalized invariants or by Freyd's (1987, 1993) hypothesis of spatiotemporal coherence, and it remains an area for additional investigation. In a follow-up study, Hubbard (1995d) presented subjects with a vertically moving target that either was presented in isolation, appeared to slide along a larger stationary object on either the left or right side of the target, or slid between larger stationary objects on both the right and left sides of the target. As in the earlier study, the magnitude of forward $M$ displacement decreased (backward $M$ displacement increased) with increases in the amount of implied friction.

In general, increasing the amount of implied friction for either horizontally (Hubbard, 1995b) or vertically (Hubbard, 1995d) moving targets resulted in smaller magnitudes of forward $M$ displacement or larger magnitudes of backward $M$ displacement. Furthermore, the ordering of displacement magnitudes as a function of friction was constant, regardless of whether $M$ displacement was primarily negative or positive, such that targets encountering less friction were displaced ahead of (i.e., they were either more positive or less negative than) targets encountering more friction. ${ }^{1}$ Importantly, observers responded as if friction were present in the display, even though the targets actually maintained a constant velocity throughout the duration of each trial, and hence did not behave as if they were being influenced by friction. Although the animations themselves might be considered unrealistic or inconsistent with friction, shifts in memory consistent with implied friction were nonetheless consistently found in the $M$ displacements. This may be interpreted as demonstrating the strength of the internalization of the friction analogue, because observers responded to the stimuli as though friction were present when in fact friction was not present.

\section{Implied Mass and Weight}

If the momentum metaphor of Finke et al. (1986) is taken literally, factors that influence physical momentum 
should also influence representational momentum. For example, the physical momentum of an object is defined as the product of that object's velocity and mass, and so the momentum metaphor predicts that both the velocity and mass of the target should influence the magnitude of displacement. Although robust effects of target velocity on representational momentum have been found (e.g., Freyd \& Finke, 1985; Hubbard \& Bharucha, 1988), effects of target mass on representational momentum have been more difficult to document. Munger and Cooper (1993) failed to find any effect of target size on representational momentum for rotating or horizontally moving targets, and Pantzer and Freyd (1989) showed observers pictures of similarly sized objects varying in implied mass (e.g., a block of Styrofoam vs. a block of brick) but failed to find any systematic effects of these conceptual manipulations of implied mass on representational momentum for rotating targets.

Hubbard (1995b) did not find any effect of target size on $M$ displacement of horizontally moving targets, but an effect of target size was found on $O$ displacement such that larger targets were displaced downward (i.e., in the direction of implied gravitational attraction) more than smaller targets. Following up on this finding, Hubbard (1995d) presented observers with vertically moving targets that were presented either in isolation or in an implied friction condition. If the target was presented in isolation, larger targets produced more positive $M$ displacement than did smaller targets; if the target was presented in an implied friction condition, larger targets led to less negative $M$ displacement than did smaller targets. Regardless of whether the vertically moving target was presented in isolation or in an implied friction condition, the remembered locations of larger targets were displaced in front of the remembered locations of smaller targets, and this pattern is consistent with the momentum metaphor and an incorporation of the principles of momentum. Hubbard (1995d) also measured displacement of stationary targets of varying sizes, and while targets of all sizes were displaced downward in the direction of implied gravitational attraction, the magnitude of downward displacement was not related to target size. This failure of target size to influence the magnitude of downward displacement for stationary targets is, however, consistent with physical principles stating that all physical objects fall at the same rate regardless of mass.

Considering data from all of the studies addressing effects of implied mass on displacement, it appears that effects of target size are found only along the axis aligned with implied gravitational attraction. Furthermore, effects of target size appear to be limited to the axis aligned with implied gravitational attraction regardless of whether the target is stationary or moving, and for moving targets, regardless of the direction of motion. An explanation for this pattern that is based on an internalized gravity analogue may be proposed: In the terrestrial environment, size tends to correlate highly with mass and mass tends to correlate highly with weight. It may be, then, that observers were responding not to the implied mass but to the implied weight. Effects of mass should influence momentum regardless of the direction of motion, but effects of weight are only felt along the axis of gravitational attraction. Indeed, given that weight is defined as the product of mass and (the acceleration due to) gravity, if observers are responding to weight rather than mass, we should observe effects of target size or implied mass only along the axis of implied gravitational attraction. This explanation can account for why Hubbard (1995b, 1995d) found that target size or implied mass influences displacement only along the axis aligned with implied gravitational attraction and why target size or implied mass did not influence representational momentum in the data of Munger and Cooper (1993) or Pantzer and Freyd (1989).

\section{Surrounding Context}

The results from the crash, bounce, and friction studies suggest that memory for the target may be influenced by the presence of nontarget context when the target directly interacts with or contacts that context. Hubbard (1993b) presented a series of studies in which the target did not directly interact with or contact the context but was still greatly influenced by the context. In the basic paradigm, a sequence of rectangular inducing stimuli and a rectangular probe were shown, and a larger square frame enclosing the inducing and probe stimuli could be visible during the presentation of the inducing stimuli or during the presentation of the probe. Frames visible during the presentation of the inducing stimuli could be either stationary, rotating at the same angular velocity and in the same direction as the inducing stimuli, or rotating at the same angular velocity and in the direction opposite to the inducing stimuli; frames visible during the presentation of the probe were stationary but could be either upright, rotated slightly backward from the orientation of the final inducing stimulus, at the same orientation as the final inducing stimulus, or rotated slightly forward from the orientation of the final inducing stimulus.

Both the context visible during the presentation of the inducing stimuli and the context visible during the presentation of the probe influenced the direction and magnitude of representational momentum of the target. If the context visible during the presentation of the inducing stimuli moved in the same direction as the target, the magnitude of representational momentum for the target increased, whereas if the context visible during the presentation of the inducing stimuli moved in the direction opposite to that of the target, the magnitude of representational momentum for the target decreased or the direction of representational momentum was reversed. If the context visible during the presentation of the probe suggested an orientation slightly beyond the orientation of the final inducing stimulus, the magnitude of representational momentum for the target increased, whereas if the context visible during the presentation of the probe suggested an orientation slightly behind the orientation of the final inducing stimulus, the magnitude of representational momentum for the target decreased or the direction of representational momentum was reversed. 
It is possible that changes in the direction or magnitude of representational momentum for the target apparently caused by changes in the context may reflect perceived changes in target identity rather than changes in the context per se. For example, if the frame was visible during the presentation of the inducing stimuli but was not visible during the presentation of the probe, the probe may have been perceived to be a different object from the one represented by the inducing stimuli. Similarly, if the frame matched the orientation of each inducing stimulus but did not match the orientation of the probe, the probe may have been perceived to be a different object from the one represented by the inducing stimuli. Such a change in identity, rather than motion of the context during induction or orientation of the context during presentation of the probe, might have accounted for the patterns in the data. However, if this identity hypothesis were true, larger differences in identity should have resulted in less representational momentum than smaller differences in identity (Kelly \& Freyd, 1987), and no effect of the direction of frame motion or orientation should have been obtained. Given that representational momentum was related more to the direction of the differences between the frame and the target orientations than to the magnitude of the differences between the frame and target orientations, the alternative hypotheses based on changes in identity were not supported.

\section{Memory Averaging and Landmark Effects}

The effect of a larger surrounding frame context on representational momentum demonstrates that nontarget context can influence the magnitude and direction of displacement in memory for the target. One way in which this influence might occur is through memory averaging - that is, through the combining of spatial information about the final position of the target with other information in memory. Within the literature on displacement two distinct senses of memory averaging have been suggested. The first sense is primarily a temporal sense in which memory for the final inducing stimulus is averaged with memory for the prior inducing stimuli. The second sense is primarily a spatial sense in which memory for the final inducing stimulus is averaged with memory for any nontarget context.

The possibility of a temporal memory averaging was initially articulated by Freyd and Johnson (1987) in their postulation of a mechanism to account for their observation that the magnitude of representational momentum peaked with relatively short retention intervals and declined or reversed with relatively long retention intervals. Freyd and Johnson suggested that this pattern could be accounted for by two different processes: a forward shift produced by representational momentum that dominated at short retention intervals, and a backward shift produced by memory averaging that dominated at long retention intervals. Memory averaging was considered to be a process whereby an observer abstracted the most "prototypical position" or "central tendency" of the set of inducing stimuli. As retention interval increased, this prototypical position or central tendency would come to reflect the remembered position of the target. When target motion was in a consistent direction, such a central tendency would necessarily be behind the third inducing stimulus, and would produce negative shifts. Inconsistent target motion (e.g., Freyd \& Johnson's 2, 1, 3 ordering of inducing stimuli) could presumably also produce negative shifts if the final inducing stimulus were presented at an extreme position; inconsistent target motion would presumably not produce systematic shifts if the final inducing stimulus were presented at a position at or near the central tendency of the set of inducing stimuli (e.g., a $1,3,2$ ordering of inducing stimuli).

The second sense of memory averaging is primarily a spatial sense in which memory for the final inducing stimulus is averaged with memory for nontarget context, and this would have the effect of shifting memory toward the nontarget context. Hubbard (1995b) found exactly this pattern, as $O$ displacement was downward when a single larger stationary object was below the target's path of motion and upward when a single larger stationary object was above the target's path of motion. Similarly, Hubbard (1995d) found that memory for a vertically moving target was displaced toward a single larger stationary object to either the left or the right side of the target. Memory averaging between the target and nontarget context was also found by Hubbard (1993b) in the increase in representational momentum when the context concurrent with the probe was rotated forward from the orientation of the final inducing stimulus and the decrease or reversal in representational momentum when the context concurrent with the probe was rotated backward from the orientation of the final inducing stimulus. In all of these examples, memory for the target was shifted toward a larger nontarget context, and such shifts are consistent with the possibility that the larger stationary objects and enclosing frames function as landmarks or reference points.

Hubbard's (1995b) data further suggest that spatial memory averaging of the target and nontarget context may combine with, or be modified by, information from other environmental invariants. For example, the downward $O$ displacement when the larger stationary object was below the target was relatively large and the upward $O$ displacement when the larger stationary object was above the target was relatively small, and this pattern may be accounted for by a combination of a landmark shift (which shifted the target toward the object) and a gravity shift (which shifted the target downward). When the landmark and gravity shifts were in the same direction (i.e., when the object was below the target), they summated, and overall downward $O$ displacement was large; when the landmark and gravity shifts were in different directions (i.e., when the object was above the target), they nearly canceled out, and overall $O$ displacement was small. If the magnitude of landmark shifts was slightly larger than the magnitude of gravity shifts, both the slight upward displacement when the object was above the target and the much larger downward displacement 
when the object was below the target may be easily accounted for. This account suggests that the magnitude of the landmark shift is not influenced by the direction of the object away from the target, a notion supported by Hubbard (1995d), who found no difference in the magnitude of $O$ displacement of vertically moving targets toward a larger stationary object as a function of whether the larger stationary object was on the left side or the right side of the target.

\section{Modularity and the Penetrability of Displacement}

The effects of context on the magnitude of representational momentum suggest that the representation of the target may be influenced or penetrated by information arising from outside the target per se, and that representational momentum does not therefore arise from modular or cognitively impenetrable processes. Indeed, the extent to which representational momentum is modular or cognitively impenetrable has been debated (e.g., Finke \& Freyd, 1989; Ranney, 1989). In order to be considered modular, a cognitive process must meet a number of requirements (see Fodor, 1983), but perhaps the most important of these requirements is that the process must be informationally encapsulated from cognitive penetration stemming from an observer's beliefs, knowledge, or expectations. Although Kelly and Freyd (1987; Freyd, 1987) suggested that representational momentum appears to meet many of the criteria for modularitysuch as rapidity (Freyd \& Johnson, 1987) and immunity to error feedback (Finke \& Freyd, 1985) - Hubbard and Bharucha (1988; Bharucha \& Hubbard, 1989; also Hubbard 1994a) suggested that representational momentumlike displacement could not result from modular processing because the direction and magnitude of displacement may be influenced by observers' expectations regarding the anticipated path of motion and the future behavior of the target.

Ranney (1989) argued that representational momentum was not modular because the effects attributable to representational momentum (and implied gravity) are smaller than the magnitude of the actual effects of momentum (and gravity) that would be exhibited by a physical target. ${ }^{2}$ In fact, Ranney appealed to the small magnitude of displacement effects to challenge not only the alleged modularity of representational momentum but also whether representational momentum represented an incorporation of the principles of momentum into the representational system. However, it may be argued that the magnitude of biases attributable to environmental invariants such as momentum and gravity should be smaller than the actual effects of momentum and gravity because a partial or smaller displacement would reflect the best compromise between the benefits of accurately extrapolating a target and the costs of inaccurately extrapolating a target that deviates from the expected course or position. Bertamini (1993) has also suggested that the relatively small effect sizes in studies of displacement need not invalidate weak versions of the phys- ical model as long as the appropriate patterns are present in the data. Thus, while Ranney is quite right in pointing out that the penetrability of representational momentum by observers' expectations suggests that the memory shifts are not modular, his further argument - that representational momentum does not exist because the effects attributed to representational momentum and other implied dynamics are small-is unwarranted.

Verfaillie and d'Ydewalle (1991) addressed the issues of penetrability and modularity by comparing representational momentum at the boundary of a periodic motion with representational momentum at the midpoint of a periodic motion or at the boundary of a nonperiodic motion. Sequences of periodic motion were created by having a rectangle oscillate around its central point (and thus change direction in a highly predictable fashion), and sequences of nonperiodic motion were created by presenting similar inducing stimulus rectangles that did not oscillate but instead maintained rotation in a consistent direction. A standard forward shift in the direction of motion was found in the nonperiodic condition and at the midpoint of a periodic motion, but when observers judged the final position of a stimulus at the boundary of a periodic motion, the magnitude of representational momentum was greatly reduced. Verfaillie and d'Ydewalle interpreted this pattern as suggesting that observers were sensitive to the higher order event patterns (i.e., the oscillations) in the inducing stimuli, and that this knowledge decreased the magnitude of representational momentum at the boundary of a periodic motion because the observers expected the inducing stimulus to reverse direction.

An even stronger case against penetrability and modularity was presented by Hubbard (1994a), who influenced the magnitude of $M$ displacement by manipulating the verbal instructions presented to observers before the target appeared. On each trial, a horizontally or vertically moving target approached an orthogonally oriented barrier and either bounced off the barrier or crashed through it. The target and barrier then simultaneously vanished, and observers positioned the cursor over the judged vanishing point of the target. Before each trial, the observer was visually presented with the cue word bounce or the cue word crash, and the probability of cue validity varied across different groups of observers. Changing the probability of cue validity did not significantly change the pattern of $M$ displacement on valid trials, but smaller forward $M$ displacements were obtained on invalid trials than on valid trials. As the overall displacement pattern for each trial type remained largely similar regardless of cue validity, at least some aspects of the displacement pattern were cognitively impenetrable and determined more by target behavior than by observers' expectations. Differences between valid and invalid trials, however, indicate some influence of observers' expectations, and so at least some aspects of the displacement pattern were cognitively penetrable. Displacement is thus not produced by purely modular pro- 
cesses; the shifts in remembered location are not informationally encapsulated, because beliefs concerning future target behavior can influence the direction and magnitude of displacement.

In the Verfaillie and d'Ydewalle (1991) and Hubbard (1994a) studies, the observers' expectations concerning target behavior were induced within the laboratory, as the observers watched the targets over the course of the experimental trials or were explicitly instructed about target behavior prior to each experimental trial. Is it possible to get cognitive penetration of the displacement pattern without changing the direction of implied or anticipated motion or without explicitly instructing observers about possible target behavior? Hubbard (1993a) examined this question by presenting listeners with auditory ascending inducing stimuli corresponding to the tonic, dominant, and octave (I, V, and $\mathrm{I}^{\prime}$ ) notes of a major scale, and the final inducing stimulus was a slightly flattened octave, an in-tune octave, or a slightly sharpened octave. If the final inducing stimulus was flat, both representational momentum and an octave schema would have shifted memory higher; if the final inducing stimulus was sharp, an impenetrable representational momentum would have shifted memory higher, but an octave schema would have shifted memory lower. In both the flat and sharp conditions, the direction of displacement was consistent with predictions based on an octave schema, thus suggesting that higher level schemata may influence the direction of displacement. Importantly, the direction of implied pitch motion was constant across tuning conditions, and so listeners had no reason to anticipate any change in the direction of target motion.

Reed and Vinson (in press) recently reported a series of studies in which they demonstrated that the magnitude of representational momentum was influenced by object- specific conceptual knowledge. In other words, target identity influenced the magnitude of displacement even when features such as shape and size were held constant across different target identities. In one experiment, representational momentum was greater for ascending motion when the target was a line drawing of a rocket than it was when the target was a line drawing of a church steeple. In a second and more convincing experiment, the target stimulus was the same for two different groups of observers, but one group was told that the target was a rocket and the second group was told that the target was a steeple. When the target ascended, observers who were told that the target was a rocket produced greater representational momentum than did observers who were told that the target was a steeple. These data suggested that observers' background schematic knowledge about rockets and steeples influenced their displacements, and offer a useful extension of Hubbard's (1994a) finding that verbal instructions (and the schematic background knowledge activated by those instructions) can influence the displacement pattern even when the physical display remains constant.

All of the studies on the possible modularity and cognitive impenetrability of displacement converge on a common conclusion: that the magnitude and direction of displacement of any given target is consistent with the higher order (schematic or conceptual) knowledge of the target and the context or event in which the target is embedded. Displacement is therefore along pathways consistent with the expectations concerning future target behavior. However, even though the magnitude and direction of displacement may differ as a function of observers' expectations, shifts of some form still occur regardless of observers' expectations. These conclusions are consistent with Finke and Freyd's (1989) suggestion that while some aspects of representational momentum (e.g., the precise path of extrapolation) may be cognitively penetrable, other aspects (e.g., the spontaneous rate of extrapolation, the continuity of the extrapolation process, and the inability to halt the extrapolation process) may be cognitively impenetrable.

\section{Displacements in Depth-Representational Momentum and Boundary Extension}

Kelly and Freyd (1987) noted that the laws of physical momentum apply equally well to targets moving in depth as to targets moving in the picture plane. However, practically all of the studies on representational momentum using visual stimuli have presented targets moving in the picture plane. One reason for this neglect of motion in depth is obvious: the CRTs on which stimuli are typically presented are oriented in the picture plane. Indeed, there is no way to directly portray changes in depth on a CRT screen, and various indirect methods (e.g., changes in monocular cues, such as the visual angle subsumed by the target) must instead be used to imply changes in depth. Additionally, the use of these indirect measures may present observers with certain ambiguities; for example, if visual angle is manipulated, are the stimuli to be interpreted as maintaining the same objective size and changing in depth or as maintaining the same objective depth and changing in size?

A preliminary investigation of displacement in depth was conducted by Hubbard (in press), who manipulated the visual angle subsumed by inducing and probe stimuli and presented black square inducing stimuli that portrayed motion either toward or away from the observer. Observers were instructed to interpret the stimuli as maintaining the same objective size and changing in depth, and examples of how visual angle changes with changes in depth were provided. Memory for slower targets was displaced backward (i.e., away from the observer for approaching motion, toward the observer for receding motion), and memory for faster targets was displaced forward (i.e., toward the observer for approaching motion, away from the observer for receding motion). The magnitude of displacement for targets moving in depth was generally smaller than the magnitude of displacement for targets moving at the same velocity horizontally in the picture plane, although for both motion in depth and motion in the picture plane faster target velocity led to more forward (less backward) displacement. One possible explanation for these patterns is that effects of mem- 
ory averaging (which would shift memory backward toward the previously presented inducing stimuli) were relatively stronger for targets moving in depth than for targets moving in the picture plane, a difference that might have occurred because the retinal coordinates of the successive inducing stimuli overlapped more for motion in depth than for motion in the picture plane.

The stimuli in Hubbard (in press) implied motion either toward or away from the observer, but displacement in depth may also be obtained in the absence of motion. Intraub and her colleagues (Intraub, Bender, \& Mangels, 1992; Intraub \& Bodamer, 1993; Intraub \& Richardson, 1989 ) documented an outward displacement in the memory for the boundaries of a static scene, referred to as boundary extension, and they attributed this outward displacement to dynamic aspects of memory and expectations produced by scene schemata. In the standard boundary extension paradigm, the observers are shown a picture of a scene, the picture is removed, and after some interval of time the observers are shown a second picture and asked whether the second picture is the same as the first. If the first and second pictures portray the same overall scene but differ in the visual angles subsumed by the objects in the scene (i.e., the portrayed distance), the observers are more likely to erroneously identify the second picture as being the same as the first picture if the second picture utilizes a wider (i.e., more panoramic) camera angle than if the second picture utilizes a narrower (i.e., more close-up) camera angle than does the first picture. Similarly, when observers draw the first picture from memory, they include elements in their drawings that logically might have been present just beyond the boundaries of the picture; also, when later shown the first picture, the observers may claim it is "closer up" than before.

In order for boundary extension of a scene to occur, each figural element in the scene must be remembered as slightly smaller than it was initially perceived to be, and so the visual angle of each figural element when that element is remembered will be smaller than the visual angle of that element when that element was initially perceived. Remembering the target as slightly smaller or as occupying a slightly smaller visual angle is geometri- cally equivalent to a displacement of the target away from the observer. Boundary extension may therefore be considered as reflecting simultaneous displacement of the figural elements within the picture (although the precise extent to which figural elements may contribute more or less than ground elements has not yet been addressed in the literature), whereas representational momentum is generally considered as reflecting displacement of just a single figural moving target (but see Finke et al., 1986) against a stationary background (but see Hubbard, 1993b). Such a shift in memory toward a smaller visual angle is also consistent with the finding that static targets are remembered as smaller than their originally perceived size, and that the psychophysical exponent for remembered area is generally less than the psychophysical exponent for perceived area (Algom, 1992; Hubbard, 1994b).

Hubbard (in press) also measured displacement in depth for black square stationary targets. Given that such targets possess neither (nonzero) momentum nor presumably evoke rich scene schemata, neither theories of representational momentum nor theories of boundary extension predicted any systematic displacement in depth. Surprisingly, when memory for three different target sizes and retention intervals of 250 and $750 \mathrm{msec}$ was examined, memory for each target was shifted toward a smaller size (i.e., away from the observer) for all target sizes after $250 \mathrm{msec}$ but toward an average of the target sizes after $750 \mathrm{msec}$. An initial displacement beyond the target followed by a memory averaging toward an average of the stimulus set is consistent with Intraub et al.'s (1992) two-component model of boundary extension and is also consistent with the initial forward displacement followed by memory averaging documented for representational momentum by Freyd and Johnson (1987). In a subsequent experiment, a similar stationary target was presented, and immediately after the target vanished, an auditory (tone) cue instructed observers to position the cursor over where either the top or the bottom edge of the target had been located. Observers were more likely to indicate a location below the top edge or above the bottom edge of the target, and this pattern is also consistent with boundary extension and memory

Table 1

Comparison of Boundary Extension (BE) and Representational Momentum (RM)

\begin{tabular}{|c|c|c|}
\hline \multirow[b]{2}{*}{ Characteristics } & \multicolumn{2}{|c|}{ Type of Displacement } \\
\hline & $\mathrm{BE}$ & RM \\
\hline Processing & dynamic, automatic & dynamic, automatic \\
\hline Direction of extrapolation & beyond the stimulus & beyond the stimulus \\
\hline Sequence & displacement, then normalization & displacement, then normalization \\
\hline Effective duration & $\begin{array}{l}\text { immediate test reveals } \\
\text { greater displacement than delayed tests }\end{array}$ & peaks at $300 \mathrm{msec}$ and then declines \\
\hline Spatial orientation & depth & picture plane \\
\hline Motion & $?$ & faster velocity leads to greater displacement \\
\hline Size & $\begin{array}{l}\text { closer (larger) target leads to greater } \\
\text { displacement }\end{array}$ & $\begin{array}{l}\text { larger target leads to greater displacement along axis } \\
\text { of gravitation attraction }\end{array}$ \\
\hline Direction of maximum displacement & $?$ & horizontal motion \\
\hline
\end{tabular}


being shifted toward a smaller visual angle and away from the observer.

The data in Hubbard (in press) suggest that representational momentum and boundary extension may be more similar than has previously been suspected, and a closer examination of the characteristics of representational momentum and boundary extension suggests that these different types of displacement share a striking number of similarities. Given the similarities listed in Table 1, it is possible that boundary extension and representational momentum may arise from either similar mechanisms or different facets of the same general displacement mechanism. In other words, representational momentum and boundary extension may be special cases of a more general and deeper underlying extrapolation process that distorts memory in directions most consistent with past experience. In representational momentum, such an extrapolation includes the next likely position just beyond the target's actual position, and in boundary extension, such an extrapolation includes the next likely elements just beyond the boundary of the scene. Although this hypothesis is a provocative one, much more empirical work will be required before the form of relationship between these types of displacement may be known with more certainty. Along these lines, it would also be useful to examine whether other environmental invariants such as gravity or friction influence boundary extension and displacement in depth in the same way that they influence displacement in the picture plane.

\section{Curvilinear Motion and Representational Centripetal Force}

All of the studies of displacement discussed to this point that have presented moving visual stimuli have used either rotating or translating targets. In the first published examination of representational momentum for visual targets undergoing curvilinear motion, Freyd and Jones (1994) presented observers with a computeranimated display in which a small ball was depicted as moving throughout nearly the entire length of a spiral tube and then exiting the tube; upon exiting, the ball was depicted as following either a spiral, curvilinear, or straight-line path. Memory for the final location of the ball was then tested. Freyd and Jones predicted that if observers had accurately internalized the correct path of motion, the largest representational momentum would occur along the (correct) straight-line path and the smallest representational momentum would occur along the (least correct) spiral path. Freyd and Jones actually found just the opposite pattern, as the largest representational momentum occurred along the spiral path and the smallest (actually negative) representational momentum occurred along the straight-line path.

Kelly and Freyd's (1987) data suggest that memory for a target traveling along a curvilinear path may be displaced along a similar curvilinear path. The presentation of curvilinear motion also offers an interesting opportunity to examine whether an additional environmentally invariant principle, centripetal force, influences memory for target location. More specifically, the motion of a target traveling along the orbit of a circle is specified by two vectors at right angles: a forward velocity $\mathbf{V}$ (corresponding to its inertia) tangent to the orbit, and an acceleration $\mathbf{A}$ toward the center of the circle. A forward displacement in the direction of inertial motion (along the $\mathbf{V}$ vector) would be consistent with representational momentum, and an inward displacement toward the center of the circle (along the A vector) would be consistent with an internalized centripetal force. Hubbard (1995c) presented observers with computer-animated target stimuli moving along a circular orbit, and after each target vanished, the observers indicated the vanishing point by positioning the cursor. As predicted, memory for the target was displaced both forward along the axis of inertial motion and inward along the axis of centripetal acceleration. The magnitude of forward displacement along the axis of inertial motion increased with increases in angular velocity of the target and also increased with increases in radius length of the orbit, whereas the magnitude of displacement along the axis of centripetal acceleration was not systematically influenced by increases in angular velocity of the target or radius length of the orbit.

The displacement patterns found by Freyd and Jones (1994) and Hubbard (1995c) are consistent with the hypothesis that the invariant principles governing circular motion have been internalized or otherwise incorporated into the representational system. One reason why memory for targets traveling a curvilinear path is shifted along both the $\mathbf{V}$ and the $\mathbf{A}$ components of curvilinear motion may be that the extrapolations that produce these shifts do not result from instantaneous processes. More specifically, just as the momentum metaphor suggests that it may not be possible to stop the extrapolation of forward motion (e.g., displacement forward along the $\mathbf{V}$ component) immediately after the target vanishes, and so representational momentum is produced, so, too, it might not be possible to halt the extrapolation of inward motion (i.e., displacement inward along the A component) immediately after the target vanishes, and so memory for the target is also displaced inward in the direction of implied centripetal force. The shifts along the $\mathbf{V}$ and A components are combined and result in an ultimate displacement of the target forward and inward.

\section{Displacement and Naive Physics}

One important element in theories of representational momentum and the effects of environmental invariants on representation is the supposition that observers have an accurate (and perhaps tacit) understanding or internalization of the physical laws governing the motion of physical objects. The veridicality of an average observer's understanding of these physical laws has been called into considerable question, however (for a review, see McCloskey, 1983). In one particularly well-discussed example, McCloskey and Kohl (1983) presented observers with a diagram depicting a spiral tube. Observers were 
told that a small ball was placed in the inner end of the tube and shot through the tube, and the observers then picked from among a number of drawings which drawing most accurately depicted the path that the ball would take after exiting the tube. The majority of observers picked a path that depicted the ball as continuing along a curved path after having exited the tube, whereas the actual physical path would have been a straight line. McCloskey and his colleagues suggested that observers may possess a naive physical theory which specifies that the tube imparts a curvilinear impetus to the ball and that this curvilinear impetus is gradually discharged after the ball exits the tube.

Although the claim that observers possess a naive theory of curvilinear impetus has recently been debated (e.g., Cooke \& Breedin, 1994a, 1994b; Ranney, 1994), the data of McCloskey and his collaborators might nonetheless initially be seen as posing a significant challenge to theories of representational momentum and related types of displacement. Freyd and Jones (1994) suggested that data from studies of naive physics do not specifically challenge theories of representational momentum because studies on naive physics focus on a different aspect of motion from that focused on by studies of representational momentum; more specifically, studies of naive physics have focused on the pathways that people choose (e.g., a straight-line or spiral pathway upon exiting the spiral tube), whereas studies of representational momentum have focused not on the choice of pathway but on the magnitude of displacement along the chosen pathway. Indeed, as discussed earlier, numerous studies have now shown that the direction and magnitude of representational momentum can be influenced by observers' beliefs or expectations, and it is certainly plausible that if observers naively believe that a ball exiting a spiral tube will continue in a spiral path, they will judge that possibility as more likely than a straight path and greater forward representational momentum along that spiral pathway will be produced.

The same processes discussed in accounting for the data of Freyd and Jones (1994) and Hubbard (1995c) may also be used to account for the naive physics data on the spiral tube problem. In the static displays used by McCloskey and his colleagues, the observers did not see the target move, and so they needed to extrapolate the trajectory that the ball would take after exiting the tube (indeed, they needed also to extrapolate the trajectory prior to exiting the tube, although the trajectory prior to exiting was totally constrained by the tube). This extrapolation of the ball's trajectory involves both forward (V) and inward (A) components similar to those observed in Freyd and Jones (1994) and Hubbard (1995c), and when the ball exits the tube, those forward and inward components are both still activated. Presumably, upon exiting the tube, the influence of the inward component ceases but the influence of the forward component does not cease. The cessation of the inward component does not happen instantaneously upon exiting, however, but requires time to implement. Immediately upon exiting the tube, the ball is not physically contained within the tube, but because of the lag in stopping the inward extrapolation, the representation of the ball is still influenced by the lingering effects of the tube; thus, extrapolation of the ball reflects the same type of path (i.e., curvilinear or spiral) that the ball traveled before exiting the tube. In essence, the apparent curvilinear impetus may reflect a type of representational centripetal force that, like representational momentum, cannot be halted instantly, but instead results in a shift of memory in the direction of the invariant force.

The explanation both of the patterns of displacement for curvilinear motion and of the naive physics spiral tube experiments in terms of forward $\mathbf{V}$ and inward $\mathbf{A}$ components of displacement suggests that these data are not in disagreement and may be accounted for within a single consistent framework. Memory for both target location and observers' extrapolations of possible target locations (i.e., trajectories) are influenced by observers' expectations concerning the future motion of the target and the forces that the observer believes may be operating on the target. Observers attempting to intercept a moving target would need to consider environmentally invariant factors such as momentum, gravity, friction, and centripetal force that might be operating on that target, and so in the real world, these types of automatic extrapolations might have been sufficiently adaptive as to have become incorporated into our representational systems. Like heuristics (e.g., Kahneman, Slovic, \& Tversky, 1982), these extrapolations are just good enough for survival in the world, but clearly break down under highly artificial laboratory conditions.

\section{Prolegomenon for a Future Network Model of Displacement}

The various theories of displacement discussed above focus primarily on momentum, and while some of the theories also address other environmental invariants, none address all of the findings and ideas that have been reviewed here. A preview of a broader network approach under construction that is capable of accounting for a wider spectrum of displacement data will be given, and the suggestions given here propose ways in which the various displacement phenomena might be more formally modeled. The suggestions made here are not meant to be a radical alternative to the ideas of Freyd, Shepard, and others; rather, the proposed network mechanisms are meant to provide a more general implementation of the previous theories. Ideally, the network suggested here, as well as any other future models of the displacement process, should include not only momentum but also other environmental invariants such as gravity, friction, and centripetal force; interactions of the target with other elements in the context; and the effects of observers' expectations regarding future target behavior.

Building upon ideas discussed by Bharucha and Hubbard (1989), Faust (1990), Grossberg and Rudd (1989), and Hubbard (1993b, 1994a, 1995b), the effects of environmental invariants, memory averaging, and observers' 
expectations may be instantiated in a fairly straightforward way within a network model. Different areas of physical space are represented by different nodes, and as a target moves across space, it traces a path of activation through the network. Nontarget context such as that provided by surfaces and objects also activates nodes corresponding to the locations of those surfaces and objects. Once a node is activated, activation spreads outward from that node along either excitatory or inhibitory pathways. The influences of environmental invariants such as momentum, gravity, friction, and centripetal force channel activation in specific directions; for example, momentum is modeled by having excitatory activation spread along the path of anticipated target motion, gravity is modeled by weighting downward excitatory connections more heavily than upward excitatory connections, friction is modeled by having inhibitory activation spread outward from nontarget context, and centripetal force is modeled by having excitatory activation spread toward the focus of a circular path. Spatial and temporal memory averaging may be accounted for by spreading activation from the nontarget context toward the target and residual activation in nodes corresponding to the previous locations of the target. Observers' expectations regarding future behavior of the target biases the network to respond in a particular way by providing additional excitatory activation to areas corresponding to the anticipated future path of the target and/or by providing inhibitory activation to areas that do not correspond to the anticipated future path of the target. Remembered location and the ultimate direction and magnitude of displacement correspond to the locus of highest activation.

Preliminary investigation suggests that many of the findings in the displacement literature might be effectively simulated within such a network approach, and construction of such a simulation is in progress. It cannot be overemphasized that in the network the ultimate direction and magnitude of displacement of a given target is an average of excitatory and inhibitory influences from many different sources. Displacement in the remembered position of the target does not arise from a single force such as momentum, but rather arises from a combination of characteristics of the target stimulus, the nontarget context, implied dynamics and other environmental contributions, and observers' expectations. The contributions from a variety of sources to the determination of the ultimate direction and magnitude of displacement within the network parallel the effects of these sources on displacement in human observers. Activations from all of these elements within the network combine in a weighted average, and the location of the network that corresponds to the highest activation value, the "center of activation," will specify the remembered location of the target.

\section{Summary and Conclusion}

When people visually perceive a moving target and that target then vanishes, memory for the final position or orientation of that target is typically shifted or dis- placed in the direction of anticipated motion. The direction and magnitude of this memory shift are influenced by a number of factors: velocity (Freyd \& Finke, 1985; Hubbard, 1990, 1995c; Hubbard \& Bharucha, 1988), acceleration (Finke et al.,1986), direction of target motion (Halpern \& Kelly, 1993; Hubbard, 1990, in press), direction of anticipated target motion (Freyd \& Jones, 1994; Hubbard, 1994a), direction of context motion (Hubbard, $1993 \mathrm{~b}$ ), direction of implied gravitational attraction (Freyd et al., 1988; Hubbard, 1990, 1995b, 1995d), constancy of target shape (Kelly \& Freyd, 1987), duration between disappearance of the target and the probing of remembered location (Freyd \& Johnson, 1987), consistency of target motion (Freyd \& Finke, 1984; Kelly \& Freyd, 1987), interaction of the target with other elements in the display (Hubbard, 1994a, 1995b), similarity of the final target to a prototypical member of a category (Kelly \& Freyd, 1987) or to a schematically proper ending (Hubbard, 1993a), object-specific conceptual knowledge of the target (Reed \& Vinson, 1994), target size or implied mass (Hubbard, 1995d), and whether motion of the target is embedded in an event hierarchy (Verfaillie \& d'Ydewalle, 1991).

By examining the findings from numerous studies, we arrive at four primary types of factors influencing displacement: stimulus characteristics, implied dynamics and environmental invariants, memory averaging, and observers' expectations regarding future target motion and any interactions of the target with nontarget context. Stimulus characteristics include properties of the target such as velocity and direction of travel. Implied dynamics include the implicit forces acting on the target such as the environmental invariants of momentum, gravity, friction, and centripetal force. Memory averaging includes tendencies for target memory to be shifted toward a nontarget context or toward an average of the final and prior positions of the target. Observers' expectations include both conscious and tacit beliefs concerning anticipated future behavior of the target, and these beliefs need not correspond with the veridical laws governing physical motion. The ultimate displacement pattern for a given target is determined by a weighted average of contributions from all of these factors.

Consistent with predictions from Freyd's (1987, 1992, 1993) ideas of dynamic representation and generalizations of Shepard's $(1984,1994)$ ideas on the internalization of environmental invariants, the data reviewed here suggest that dynamic aspects of memory corresponding to environmental invariants can influence or displace memory. Although displacement does not depend upon the activation of object-specific conceptual knowledge or scene structure (and thus may be considered to be basic or intrinsic to representation), displacement is transparent to an observer's beliefs, knowledge, or expectations, even if such information is not explicitly told to the observer during the experimental session but instead manifests through tacit or implicit knowledge (and thus may be considered cognitively penetrable). Such influ- 
ences reflect the history of learning of the individual and of the species, because while human evolution may have internalized the general practical implications of momentum, gravity, friction, centripetal force, and mass, an individual's own experiences prior to and during the experimental session can modify the way in which those influences are manifested. Consistent with the speculations of Finke and Freyd (1989), there is strong evidence for both mental extrapolation and flexibility in the precise pathway of such an extrapolation.

The ideas presented here also go beyond those suggested by Freyd and Shepard, however. In addition to examining a broader range of environmental invariants and including influences of observers' beliefs or expectations on memory for location, the ideas discussed here challenge arguments that the level of influence of the universe upon our representational system is geometric and that the incorporated features of the universe are kinematic. A purely kinematic explanation would focus only on the geometrical constraints and would not support any role for physical dynamics or forces, but the influence of implied friction, centripetal force, and gravity (weight) all support the incorporation (or at least the acknowledgment) of force as well as geometry. Indeed, the data are more consistent with a dynamic model than with a purely geometric or kinematic model. Finally, the approach here continues in the spirit of Shepard's (1984) attempts to reconcile Gibsonian and representational approaches, in that it acknowledges roles for both general environmental invariants and specific individual beliefs and experiences.

\section{REFERENCES}

Algom, D. (1992). Memory psychophysics: An examination of its perceptual and cognitive prospects. In D. Algom (Ed.), Psychophysical approaches to cognition (pp. 441-513). New York: Elsevier, NorthHolland.

BERTAMINI, M. (1993). Memory for position and dynamic representations. Memory \& Cognition, 21, 449-457.

Bharucha, J. J., \& HUBbard, T. L. (1989). Anticipated collision along an oblique path: Effects on judged displacement. Unpublished manuscript.

CoOKE, N. J., \& BreEdiN, S. D. (1994a). Constructing naive theories of motion on the fly. Memory \& Cognition, 22, 474-493.

Cooke, N. J., \& BreEdiN, S. D. (1994b). Naive misconceptions of Cooke and Breedin's research: Response to Ranney. Memory \& Cognition, 22, 503-507.

FAUST, M. (1990). Representational momentum: A dual process perspective. Unpublished doctoral dissertation, University of Oregon, Eugene.

FINKE, R. A., \& FREYD, J. J. (1985). Transformations of visual memory induced by implied motions of pattern elements. Journal of Experimental Psychology: Learning, Memory, \& Cognition, 11, 780-794.

FinKE, R. A., \& FREYD, J. J. (1989). Mental extrapolation and cognitive penetrability: Reply to Ranney and proposals for evaluative criteria. Journal of Experimental Psychology: General, 118, 403-408.

Finke, R. A., Freyd, J. J., \& ShyI, G. C. W. (1986). Implied velocity and acceleration induce transformations of visual memory. Journal of Experimental Psychology: General, 115, 175-188.

FinKE, R. A., \& Shyı, G. C. W. (1988). Mental extrapolation and representational momentum for complex implied motions. Journal of
Experimental Psychology: Learning, Memory, \& Cognition, 14, $112-120$

FODOR, J. A. (1983). The modularity of mind. Cambridge, MA: MIT Press.

FreYd, J. J. (1983). The mental representation of movement when static stimuli are viewed. Perception \& Psychophysics, 33, 575-581.

FREYD, J. J. (1987). Dynamic mental representations. Psychological Review, 94, 427-438.

FREYD, J. J. (1992). Dynamic representations guiding adaptive behavior. In F. Macar, V. Pouthas, \& W. J. Friedman (Eds.), Time, action, and cognition: Towards bridging the gap (pp. 309-323). Dordrecht: Kluver.

FrEYD, J. J. (1993). Five hunches about perceptual processes and dynamic representations. In D. Meyer \& S. Kornblum (Eds.), Attention and performance XIV: Synergies in experimental psychology, artificial intelligence, and cognitive neuroscience (pp. 99-119). Cambridge, MA: MIT Press.

FREYD, J. J., \& FinKE, R. A. (1984). Representational momentum. Journal of Experimental Psychology: Learning, Memory, \& Cognition, 10, 126-132.

FREYD, J. J., \& FinKE, R. A. (1985). A velocity effect for representational momentum. Bulletin of the Psychonomic Society, 23, 443446.

FREYD, J. J., \& Johnson, J. Q. (1987). Probing the time course of representational momentum. Journal of Experimental Psychology: Learning, Memory, \& Cognition, 13, 259-269.

FREYD, J. J., \& JoNES, K. T. (1994). Representational momentum for a spiral path. Journal of Experimental Psychology: Learning, Memory, \& Cognition, 20, 968-976.

FreYd, J. J., KelLy, M. H. \& DeKay, M. L. (1990). Representational momentum in memory for pitch. Journal of Experimental Psychology: Learning, Memory, \& Cognition, 16, 1107-1117.

Freyd, J. J., Pantzer, T. M., \& Cheng, J. L. (1988). Representing statics as forces in equilibrium. Journal of Experimental Psychology: General, 117, 395-407.

FutTERWEIT, L. R., \& BeILIN, H. (1994). Recognition memory for movement in photographs: A developmental study. Journal of Experimental Child Psychology, 57, 163-179.

GrossBerg, S., \& RuDD, M. E. (1989). A neural architecture for visual motion perception: Group and element apparent motion. Neural Networks, 2, 421-450

HALPERN, A. R., \& KeLLY, M. H. (1993). Memory biases in left versus right implied motion. Journal of Experimental Psychology: Learning, Memory, \& Cognition, 19, 471-484.

HubBard, T. L. (1990). Cognitive representation of linear motion: Possible direction and gravity effects in judged displacement. Memory \& Cognition, 18, 299-309.

HubBard, T. L. (1993a). Auditory representational momentum: Musical schemata and modularity. Bulletin of the Psychonomic Society, 31, 201-204

HUbBard, T. L. (1993b). The effect of context on visual representational momentum. Memory \& Cognition, 21, 103-114.

HubBaRD, T. L. (1994a). Judged displacement: A modular process? American Journal of Psychology, 107, 359-373.

Hubbard, T. L. (1994b). Memory psychophysics. Psychological Research/Psychologische Forschung, 56, 237-250.

HuBbard, T. L. (1995a). Auditory representational momentum: Surface form, velocity, and direction effects. American Journal of Psychology, 108, 255-274.

HuBBaRD, T. L. (1995b). Cognitive representation of motion: Evidence for representational friction and gravity analogues. Journal of Experimental Psychology: Learning, Memory, \& Cognition, 21, 1-14.

HUBBARD, T. L. (1995c). Representational momentum, centripetal force, and curvilinear impetus. Manuscript submitted for publication.

Hubbard, T. L. (1995d). Target size and displacement along the axis of implied gravitational attraction. Manuscript submitted for publication.

HubBard, T. L. (in press). Displacement in depth: Representational momentum and boundary extension. Psychological Research/Psy. chologische Forschung. 
HubBard, T. L., \& Bharucha, J. J. (1988). Judged displacement ín apparent vertical and horizontal motion. Perception \& Psychophysics, 44, 211-221.

Intraub, H., Bender, R. S., \& Mangels, J. A. (1992). Looking at pictures but remembering scenes. Journal of Experimental Psychology: Learning, Memory, \& Cognition, 18, 180-191.

Intraub, H., \& Bodamer, J. L. (1993). Boundary extension: Fundamental aspect of pictorial representation or encoding artifact? Journal of Experimental Psychology: Learning, Memory, \& Cognition, 19, 1387-1397.

INTRAUB, H., \& Richardson, M. (1989). Wide-angle memories of close-up scenes. Journal of Experimental Psychology: Learning, Memory, \& Cognition, 15, 179-187.

Kahneman, D., Slovic, P., \& Tversky, A. (1982). Judgment under un certainty: Heuristics and biases. New York: Cambridge University Press.

KeLLY, M. H., \& FrEYD, J. J. (1987). Explorations of representational momentum. Cognitive Psychology, 19, 369-401.

McCloskey, M. (1983). Naive theories of motion. In D. Gentner \& A. L. Stevens (Eds.), Mental models (pp. 299-324). Hillsdale, NJ: Erlbaum.

MCClosKey, M., \& KoHL, D. (1983). Naive physics: The curvilinear impetus principle and its role in interactions with moving objects. Journal of Experimental Psychology: Learning, Memory, \& Cognition, 9, 146-156.

MungER, M. P., \& COOPER, L. A. (1993, November). What is represented in representational momentum? Poster presented at the meeting of the Psychonomic Society, Washington, DC.

PANTZER, T. M., \& Freyd, J. J. (1989). Searching for a mass effect for representational momentum. Unpublished manuscript.

RANNEY, M. (1989). Internally represented forces may be cognitively penetrable: Comment on Freyd, Pantzer, and Cheng (1988). Journal of Experimental Psychology: General, 118, 399-402.

RANNEY, M. (1994). Relative consistency and subjects' "theories" in domains such as naive physics: Common research difficulties illustrated by Cooke and Breedin. Memory \& Cognition, 22, 494-502.

ReED, C. L., \& Vinson, N. G. (in press). Conceptual effects on representational momentum. Journal of Experimental Psychology: Human Perception \& Performance.

SHEPARD, R. N. (1984). Ecological constraints on internal representation: Resonant kinematics of perceiving, imaging, thinking, and dreaming. Psychological Review, 91, 417-447.

SHEPARD, R. N. (1994). Perceptual-cognitive universals as reflections of the world. Psychonomic Bulletin \& Review, 1, 2-28.

SHIFFRAR, M. M., \& SHePARD, R. N. (1991). Comparison of cube rotations around axes inclined relative to the environment or to the cube. Journal of Experimental Psychology: Human Perception \& Performance, 17, 44-54
Verfaillie, K., \& d'Ydewalle, G. (1991). Representational momentum and event course anticipation in the perception of implied periodical motions. Journal of Experimental Psychology: Learning. Memory, \& Cognition, 17, 302-313.

White, H., Minor, S. W., Merrell, J., \& SMith, T. (1993). Representational-momentum effects in the cerebral hemispheres. Brain \& Cognition, 22, 161-170

\section{NOTES}

1. It might be objected that the negative $M$ displacements obtained in some conditions suggest that neither representational momentum nor representational friction exists. Such an objection would claim that neither physical momentum nor physical friction would actually move a physical object backward, and so there is no reason to suggest that either representational momentum or representational friction would displace memory for the target backward. However, such a viewpoint ignores the possibility (1) that either representational momentum or representational friction may be combined with or modified by other invariants or information in memory, and (2) that the direction of action of a single environmental invariant need not be in the same direction as the ultimate displacement for that target. To make these points more intuitive, we may consider representational momentum and representational friction as vectors acting upon the target. The vector for representational momentum would point forward and the vector for representational friction would point backward, and these different directions are consistent with the findings that increases in implied momentum (with friction held constant) lead to increases in forward displacement, and that increases in implied friction (with velocity held constant) lead to decreases in forward displacement. The individual effects of momentum and friction thus appear to operate in opposing directions, and the remembered location may reflect an average of these two effects. If the target either has a high velocity or encounters a small amount of friction, the momentum vector would be longer than the friction vector and the ultimate displacement would be forward; if the target either has a slow velocity or encounters a large amount of friction, the momentum vector would be smaller than the friction vector and the ultimate displacement would be backward.

2. Ranney's comments concerning effect sizes could also be extended to the subsequent research on effects of implied friction and implied mass because the magnitude of effects for those factors is also considerably smaller than the magnitude of the actual effects of friction and mass that would be exhibited by a physical target.

(Manuscript received July 14, 1994; revision accepted for publication January 24,1995 .) 